\title{
ADAPTACIÓN DEL CUESTIONARIO DE ESTILOS DE APRENDIZAJE EN ESTUDIANTES DE 4TO, 5TO DE SECUNDARIA Y UNIVERSITARIOS DE LOS PRIMEROS CICLOS, EN UNA MUESTRA PILOTO
}

Adaptation of the learning styles questionnaire in 4th, 5th grade high school students and university students of the first semester, in a pilot sample

\author{
Susan Salazar Díaz* \\ Mirella Llanos Moreno**
}

\begin{abstract}
Resumen
Investigación de tipo descriptivo, realizada en una muestra de 150 estudiantes cursando el 4to.o 5 to. de secundaria o los primeros ciclos de estudios universitarios. El objetivo de la investigación fue determinar las características psicométricas del cuestionario de estilos de aprendizaje de Morey - Alonso adaptado al Perú por Zavala. Como resultado del estudio se eliminaron 15 items de la prueba original, hallándose índices confiables.
\end{abstract}

Palabras clave: Adaptación psicométrica, cuestionario de estilos de aprendizaje, estudiantes secundaria.

\begin{abstract}
Descriptive-type research, carried out in a sample of 150 students in the 4th or 5th high school grade or the first semesters of university studies. The objective of the research was to determine the psychometric characteristics of Morey-Alonso's questionnaire of learning styles adapted to Peru by Zavala. As a result of the research, 15 items in the original test were deleted, and reliable indices were found.
\end{abstract}

Keywords: psychometric adaptation, learning styles questionnaire, adaptation

\footnotetext{
* Estudiante del XII ciclo de Psicología en la Universidad Femenina del Sagrado Corazón. susancarolina.sd@hotmail.com

** Estudiante del XII ciclo de Psicología en la Universidad Femenina del Sagrado Corazón.
} 


\section{MARCO TEÓRICO}

Para definir las estrategias de aprendizaje según la visión constructivista Pimineta (2008) lo define como un proceso activo en el que cada alumno construye nuevas ideas a partir de los conocimientos previos y las experiencias de cada uno. Así, cada persona selecciona y transforma información de acuerdo a sus propios esquemas cognitivos, lo que denomina estructuraesquema, modelo mental. (Pimineta, 2008) No todos aprendemos de la misma manera ni al mismo ritmo. Cada individuo otorga un significado y una organización diferente a la información que recibe, lo que le permite ir más allá. Al respecto: "El aprendizaje significativo ocurre cuando el sujeto elabora la información que recibe, la relaciona de modo sustantivo con lo que ya sabe y la integra a sus propia estructura cognoscitiva". (Meza y Lazarte, 2007, p. 17)

El proceso de aprendizaje según el enfoque constructivista, pasa por varias fases que obligan al individuo a participar en él de forma activa, no se trata sólo de recibir información pasivamente, sino de relacionar esa información con lo que ya sabemos y ser capaces de integrarla creando así una nueva estructura, enriqueciéndonos en el proceso. Calero (2009) refiere que una estrategia de aprendizaje implica un dominio de una serie de procedimientos y componentes que contienen técnicas que se combinan de forma deliberada para alcanzar un determinado propósito de aprendizaje.

Estas técnicas y procedimientos necesariamente requieren para su eficiencia el entrenamiento de actitudes como la cooperación, la creatividad y la criticidad, lo que conllevaría a un buen proceso de aprendizaje.

Calero (2009) refiere que para la actitud cooperativa es necesario afirmar nuestro yo en la interacción con los demás, mediante la práctica del trabajo en grupo. La solidaridad, el respeto mutuo y el diálogo permanente. Para la creatividad, quien aprende debe esforzarse en dar apertura a su sensibilidad, al trabajo, a la flexibilidad y originalidad en el pensar y en el actuar, Para la criticidad, desarrollar la reflexión, el espíritu crítico para entender el mundo y operar sobre él.
Otra estrategia a usar en el proceso de aprendizaje es la motivación como una condición básica de dicho proceso. Meza y Lazarte (2007) indica al respecto:

Una persona con buen nivel de motivación atiende mejor, relaciona mejor la información; tiene más deseos de recordarla, está más dispuesta a usar estrategias para mejorar su aprendizaje. Mientras que una persona con bajo nivel de motivación no puede procesar adecuadamente la información, no aprende o aprende débilmente. (p. 36)

Para Suarez (2009), el aprendizaje significativo pasa necesariamente por la motivación como la fuente para enriquecer las estrategias usadas en el proceso de aprender. Se trata de motivar y enseñar a pensar y actuar a través de contenidos significativos y contextualizados.

En este contexto, el profesor cumple un rol importante también ya que "debe ayudar al niño a descubrir qué contenidos son significativos o importantes para él, “(Calero, 2008, p. 121).

Otro punto importante es la autoestima como factor que condiciona el aprendizaje. Ausubel, citado por Calero (2008), al respecto indica que la adquisición de nuevas ideas y aprendizaje está subordinada a nuestras actitudes básicas; de estas depende que los umbrales de la percepción estén abiertos o cerrados, que una red interna dificulte o favorezca la integración de la estructura mental del alumno, que se generen energías más intensas de atención y concentración. Las redes internas nos hablan de la autoimagen que tiene cada alumno. Sólo en la medida en que el alumno se sienta capaz de realizar alguna tarea a través de una autoestima.

\section{FORMULACIÓN DEL ESTUDIO}

¿Cuáles son las características psicométricas del cuestionario de estilos de aprendizajes en alumnos de $4^{\circ}, 5^{\circ}$ de secundaria y primeros ciclos de universidades de Lima metropolitana? 


\section{FINALIDAD DE LA INVESTIGACIÓN}

- Adaptar el instrumento de estilo de aprendizaje en los alumnos de $4^{\circ}, 5^{\circ}$ de secundaria y primeros ciclos de universidades en una muestra piloto de Lima Metropolitana.

- Calcular las características psicométricas del el instrumento de estilo de aprendizaje en los alumnos de $4^{\circ}, 5^{\circ}$ de secundaria y primeros ciclos de universidades en una muestra piloto de Lima Metropolitana.

\section{METODOLOGÍA}

\section{- Tipo de estudio:}

Este estudio enmarca dentro de los estudios descriptivos.

\section{- Variable:}

Estilos de aprendizaje

\section{- Participantes:}

La muestra fue conformada por 150 alumnos de $4^{\circ}, 5^{\circ}$ de secundaria y primeros ciclos de universidades de Lima metropolitana.

\section{- Instrumentos de evaluación:}

El instrumento que se utilizó para la evaluación es el cuestionario de Estilos de Aprendizaje de los autores Morey - Alonso adaptado al Perú por Zavala.

\section{- Procedimiento:}

Para la adaptación del cuestionario se realizó tres estudios pilotos, con el fin de calcular la confiabilidad del cuestionario, resultando un total de 48 ítems confiables.

\section{RESULTADOS}

Tabla 1.

Alfa para cada Dimensión de los Estilos de Aprendizaje

\begin{tabular}{lcl}
\hline Estilos & Alfa de Crombach & $\mathbf{N}^{\circ}$ \\
\hline Activo & .720 & 12 \\
Reflexivo & .786 & 11 \\
Teórico & .753 & 14 \\
pragmático & .634 & 11 \\
\hline
\end{tabular}

Nota: piloto 2011

En la tabla 1 se puede observar que para los estilos de aprendizaje los alfas de Crombach son altos. Estos resultados fueron obtenidos tras la aplicación de una prueba piloto; a la que se le realizó algunas modificaciones. 
Tabla 2.

Cuestionario para el estilo de aprendizaje de cada ítem

\begin{tabular}{|c|c|c|c|c|c|c|c|c|c|c|c|}
\hline \multicolumn{4}{|c|}{ Activo } & \multicolumn{3}{|l|}{ Reflexivo } & \multicolumn{3}{|l|}{ Teórico } & \multicolumn{2}{|l|}{ Pragmático } \\
\hline$\stackrel{\mathscr{\mathscr { E }}}{\stackrel{\mathscr{E}}{\underline{\underline{D}}}}$ & $\begin{array}{l}\text { Correlación } \\
\text { elemento- } \\
\text { total } \\
\text { corregida }\end{array}$ & Alfa & $\begin{array}{l}\stackrel{\mathscr{D}}{\mathbb{E}} \\
. \underline{\underline{I}}\end{array}$ & $\begin{array}{l}\text { Correlación } \\
\text { elemento- } \\
\text { total } \\
\text { corregida }\end{array}$ & Alfa & $\underset{\stackrel{\mathscr{E}}{\Phi}}{\stackrel{\mathscr{E}}{ \pm}}$ & $\begin{array}{l}\text { Correlación } \\
\text { elemento- } \\
\text { total } \\
\text { corregida }\end{array}$ & Alfa & $\begin{array}{l}\stackrel{\mathscr{E}}{\Phi} \\
\stackrel{\Phi}{\Phi}\end{array}$ & $\begin{array}{l}\text { Correlación } \\
\text { elemento- } \\
\text { total } \\
\text { corregida }\end{array}$ & Alfa \\
\hline 1 & .332 & .705 & 13 & .389 & .775 & 24 & .483 & .726 & 38 & .272 & .616 \\
\hline 2 & .309 & .707 & 14 & .653 & .745 & 25 & .425 & .732 & 39 & .285 & .617 \\
\hline 3 & .343 & .703 & 15 & .420 & .771 & 26 & .313 & .745 & 40 & .313 & .609 \\
\hline 4 & .643 & .660 & 16 & .374 & .778 & 27 & .351 & .741 & 41 & .238 & .621 \\
\hline 5 & .474 & .684 & 17 & .549 & .756 & 28 & .530 & .722 & 42 & .318 & .606 \\
\hline 6 & .322 & .706 & 18 & .420 & .771 & 29 & .191 & .754 & 43 & .373 & .597 \\
\hline 7 & .239 & .717 & 19 & .354 & .779 & 30 & .334 & .742 & 44 & .283 & .614 \\
\hline 8 & .205 & .718 & 20 & .299 & .784 & 31 & .300 & .747 & 45 & .230 & .623 \\
\hline 9 & .412 & .693 & 21 & .557 & .756 & 32 & .280 & .748 & 46 & .272 & .617 \\
\hline 10 & .276 & .712 & 22 & .491 & .764 & 33 & .312 & .744 & 47 & .274 & .617 \\
\hline 11 & .385 & .697 & 23 & .375 & .776 & 34 & .562 & .720 & 48 & .374 & .593 \\
\hline \multirow[t]{3}{*}{12} & .282 & .711 & & & & 35 & .320 & .743 & & & \\
\hline & & & & & & 36 & .434 & .732 & & & \\
\hline & & & & & & 37 & .289 & .746 & & & \\
\hline
\end{tabular}

Nota: Piloto 2011

Se observa las correlaciones alfas para cada una de las dimensiones de los estilos de aprendizaje.

En el estilo activo, se puede observar que la correlación más alta es para el ítem cinco con un valor de 474, y si este fuese eliminado, el alfa total de la dimensión disminuirá .684, por otro lado el ítem con correlación más baja es para el ítem 12 que es de .282 que disminuirá en un .711.

En el estilo reflexivo se puede observar que la correlación más alfa es para el ítem veintidós con un valor de .491 si fuese eliminado el alfa total de la dimensión disminuiría en .764, por otro lado el ítem 19 con correlación más baja es el diecinueve es de .354 y disminuiría en .779.

En el estilo teórico se puede observar que la correlación más alta es para el ítem treinta cuatro con un valor .562 y si fuese eliminado el alfa total de la dimensión disminuiría en .720. Así mismo, el ítem con correlación más baja es el veintinueve es de .191 y disminuiría en .754.En el pragmático se puede observar que la correlación más alta es para el ítem cuarenta y ocho con un valor de .374 y si fuese eliminado el alfa total de la dimensión disminuirá en . 593, por otro lado el ítem con correlación más baja es el cuarenta y uno y es de .238 y disminuirá .621 .

En conclusión, podemos decir que los ítems correspondientes a la categoría reflexivo y pragmático, muestran una menor confiabilidad con respecto a su correspondencia. Por otro lado en los ítems correspondientes a la categoría Activo y Teórico presentan un alto índice de confiabilidad. 
Tabla 3.

Cuestionario para el estilo de aprendizaje de cada ítem

\begin{tabular}{|c|c|c|c|c|c|c|c|c|c|c|c|}
\hline \multicolumn{4}{|c|}{ Activo } & \multicolumn{3}{|c|}{ Reflexivo } & \multicolumn{3}{|c|}{ Teórico } & \multicolumn{2}{|c|}{ Pragmático } \\
\hline Ítems & $\begin{array}{l}\text { Correlación } \\
\text { elemento- } \\
\text { total } \\
\text { corregida }\end{array}$ & Alfa & İtems & $\begin{array}{l}\text { Correlación } \\
\text { elemento- } \\
\text { total } \\
\text { corregida }\end{array}$ & Alfa & Ítems & $\begin{array}{l}\text { Correlación } \\
\text { elemento- } \\
\text { total } \\
\text { corregida }\end{array}$ & Alfa & İtems & $\begin{array}{l}\text { Correlación } \\
\text { elemento- } \\
\text { total } \\
\text { corregida }\end{array}$ & Alfa \\
\hline $\begin{array}{l}1 \\
2 \\
3 \\
4 \\
5 \\
6 \\
7 \\
8 \\
9 \\
10 \\
11 \\
12\end{array}$ & $\begin{array}{l}.437 \\
.437 \\
.437 \\
.411 \\
.437 \\
.480 \\
.489 \\
.437 \\
.635 \\
.303 \\
.227 \\
.267\end{array}$ & $\begin{array}{l}.704 \\
.724 \\
.741 \\
.708 \\
.705 \\
.701 \\
.697 \\
725 \\
.682 \\
.722 \\
.731 \\
.726\end{array}$ & $\begin{array}{l}13 \\
14 \\
15 \\
16 \\
17 \\
18 \\
19 \\
20 \\
21 \\
22 \\
23\end{array}$ & $\begin{array}{l}.323 \\
.339 \\
.671 \\
.712 \\
.758 \\
.556 \\
.019 \\
.036 \\
.529 \\
.741 \\
.432\end{array}$ & $\begin{array}{l}.797 \\
.794 \\
.759 \\
.751 \\
.753 \\
.773 \\
.823 \\
.827 \\
.775 \\
.748 \\
.785\end{array}$ & $\begin{array}{l}24 \\
25 \\
26 \\
27 \\
28 \\
29 \\
30 \\
31 \\
32 \\
33 \\
34 \\
35 \\
36 \\
37\end{array}$ & $\begin{array}{l}.576 \\
.576 \\
-.096 \\
.215 \\
.542 \\
.558 \\
.227 \\
.400 \\
.302 \\
.489 \\
.475 \\
.303 \\
.441 \\
.288\end{array}$ & $\begin{array}{l}.686 \\
.686 \\
.686 \\
.732 \\
.686 \\
.694 \\
.731 \\
.709 \\
.722 \\
.703 \\
.702 \\
.721 \\
.705 \\
.722\end{array}$ & $\begin{array}{l}38 \\
39 \\
40 \\
41 \\
42 \\
43 \\
44 \\
45 \\
46 \\
47 \\
48\end{array}$ & $\begin{array}{l}.146 \\
.238 \\
.482 \\
.417 \\
-.006 \\
.522 \\
.322 \\
.367 \\
.246 \\
.282 \\
.372\end{array}$ & $\begin{array}{l}.651 \\
.637 \\
.597 \\
.603 \\
.685 \\
.583 \\
.621 \\
.612 \\
.636 \\
.629 \\
.610\end{array}$ \\
\hline
\end{tabular}

Nota: Piloto 2012

Se observa la correlación de ítems escala total y el alfa de cronBach si el ítem es eliminado, se aprecia que la mayoría de los ítems son confiables, si en el ensayo los ítems: 19 con un valor de -.019, el ítem 20 con un valor de .036, el ítem 26 con un valor de -.096 y el ítem 42 con un valor de -.006, no cumplen con los niveles mínimos de un valor de .20. Sin embargo se consideran para la aplicación de la muestra total. 
Tabla 4

Categoría de Calificación por cada dimensión de los Estilos de Aprendizaje

\begin{tabular}{crrrr}
\hline $\begin{array}{c}\text { Estilos Aprendizaje } \\
\text { /Percentil }\end{array}$ & Activo & Reflexivo & Teórico & Pragmático \\
\hline Alto & 27 - Más & 19 - Más & 24 - Más & 21 - Más \\
Medio & $19-26$ & $14-18$ & $18-23$ & $15-20$ \\
Bajo & Menor - 18 & Menor - 13 & Menor - 17 & $11-14$ \\
\hline
\end{tabular}

Nota: piloto 2012

Los resultados de la tabla de percentiles indican que los percentiles bajos para el estilo Activo se encontrarían entre los valores menores a 18; los valores medios entre el 19 y 26 ; y los valores altos mayores a 27.

Para el estilo Reflexivo los valores bajos se ubicarían entre los menores a 13; los valores medios se encuentran entre 14 y 18 ; y los valores altos mayores a 19.
Para el estilo Teórico los valores bajos se encuentran entre la frecuencia menor a 17 ; los valores medios entre la frecuencia 18 y 23 ; y los valores altos mayores a 24 .

Para el estilo Pragmático de aprendizaje, los valores bajos oscilan entre el percentil 11 y 14 ; los valores medios entre 15 y 20 ; y los valores altos mayores a 21 .

Tabla 5

Norma percentiles para el cuestionario de estilos de aprendizaje.

\begin{tabular}{ccccc}
\hline & Activo & Reflexivo & Teórico & Pragmático \\
\hline Validos & 115 & 115 & 115 & 115 \\
99 & 12.1600 & 11.0000 & 13.0000 & 11.0000 \\
95 & 14.0000 & 11.0000 & 14.0000 & 12.0000 \\
90 & 16.0000 & 12.0000 & 15.0000 & 13.0000 \\
85 & 17.0000 & 12.0000 & 16.0000 & 13.0000 \\
80 & 17.2000 & 12.0000 & 17.0000 & 14.0000 \\
75 & 18.0000 & 13.0000 & 17.0000 & 14.0000 \\
70 & 19.0000 & 13.0000 & 17.8000 & 15.0000 \\
65 & 20.0000 & 13.0000 & 18.0000 & 16.0000 \\
60 & 20.0000 & 14.0000 & 18.4000 & 16.0000 \\
55 & 21.0000 & 14.0000 & 19.0000 & 17.0000 \\
50 & 22.0000 & 15.0000 & 20.0000 & 17.0000 \\
45 & 22.0000 & 15.0000 & 21.0000 & 17.0000 \\
40 & 23.6000 & 16.0000 & 22.0000 & 18.0000 \\
35 & 24.0000 & 17.0000 & 22.0000 & 19.0000 \\
30 & 25.0000 & 17.0000 & 23.0000 & 19.2000 \\
25 & 26.0000 & 18.0000 & 23.0000 & 20.0000 \\
20 & 26.8000 & 19.0000 & 24.0000 & 20.0000 \\
15 & 28.0000 & 20.0000 & 25.0000 & 21.0000 \\
10 & 29.0000 & 22.0000 & 27.0000 & 23.0000 \\
5 & 31.0000 & 25.2000 & 31.4000 & 25.0000 \\
1 & 33.0000 & 30.6800 & 38.2000 & 27.0000 \\
\hline
\end{tabular}

Nota: piloto 2012 
Se observa el mayor puntaje para los estilos teórico y activo.

Respecto al estilo activo, podemos decir que los puntajes para el nivel alto oscilan entre 12 y 17 puntos, mientras que el puntaje para el nivel medio oscila entre 18 y 25 puntos y para el nivel bajo oscilan entre 26 y 33 puntos.

Respecto al estilo reflexivo, podemos decir que los puntajes para el nivel alto oscilan entre 11 y 12 puntos, mientras que los puntajes para el nivel medio oscila entre 13 y 17 puntos y para el nivel bajo oscilan entre 18 y 31 puntos.

Respecto al estilo teórico, podemos decir que los puntajes para el nivel alto oscilan entre 13 y 17 puntos, mientras que los puntajes para el nivel medio oscila entre 18 y 23 puntos y para el nivel baja oscilan entre 24 y 38 puntos.

Respecto al estilo pragmático, podemos decir que los puntajes para el nivel alto oscilan entre 11 y 14 puntos, mientras que los puntajes para el nivel medio oscila entre 15 y 20 puntos y para el nivel bajo oscilan entre 21 y 27 puntos.

\section{CONCLUSIONES}

- Los resultados indican que después de la primera aplicación de la prueba piloto, el instrumento tuvo que ser modificado, se eliminaron 15 ítems del documento original, siendo los ítems más inestables los correspondientes a los estilos reflexivos y pragmáticos.

- Podemosdecir,queenlosítems correspondientes a los Reflexivos y Pragmáticos, tenían una mayor inestabilidad, algo significativa, con respecto a su correspondencia, en cambio se ve en los ítems correspondientes a Activos y Teóricos, sobresaliendo este último, se maneja un índice más confiable.

- En cuanto a la identificación de frecuencias encontramos que los estilos Activo y Teórico muestran las frecuencias más altas, mientras que los estilos Reflexivo y Pragmático muestran las frecuencias más bajas.

- En lo que respecta a la identificación de correlaciones, se encontró que los estilos Activo y Reflexivo, así como Activo y Teórico muestran una correlación significativa inversa y los estilos Activo y Pragmático muestran una correlación significativa directa.

\section{REFERENCIAS}

Calero, M. (2008) Constructivismo Pedagógico: teorías y aplicaciones básicas. Lima: Ed. Alfa Omega.

Calero, M. (2009) Aprendizajes sin límites: Constructivismo. Lima: Ed. Alfa Omega

Meza y Lazarte, A. (2007) Manual de Estrategias para el Aprendizaje Autónomo y Eficaz. Lima: Ed. Universitaria Ricardo Palma.

Pimienta, J. (2008) Constructivismo: Estrategias para Aprender a Aprender. México: Ed. Pearson Educación.

Suarez, R. (2009) La Educación, estrategias de Enseñanza aprendizaje, Teorías Educativas. México: Ed. Trillas. 


\section{CUESTIONARIO DE \\ ESTILOS DE APRENDIZAJE}

\section{DATOS INFORMATIVOS}

Nombre

Sexo

Masculino

Femenino

(Marque con un aspa)

Edad

Universidad

Ciclo

Fecha

\section{INSTRUCCIONES PARA RESOLVER EL CUESTIONARIO}

- Este cuestionario ha sido diseñado para identificar su estilo preferido de aprendizaje. No es un test de inteligencia ni de personalidad.

- No hay respuestas correctas o erróneas. Será útil en la medida que sea sincero/a en sus respuestas. No le ocupará más de 15 minutos

- Marcar con un aspa el grado en que Ud. se encuentra de acuerdo o en desacuerdo con la aseveración, y si ninguna de las anteriores se aplica a su respuesta marque indiferente.

- Por favor, conteste todos los ítems. Muchas gracias.

\section{CUESTIONARIO (CHAEA)}

\begin{tabular}{lllll}
\hline $\mathbf{N}^{\circ}$ & ÍTEMS & De acuerdo & Indiferente & En desacuerdo
\end{tabular}

1. Muchas veces actuó sin mirar las consecuencias.

2. Creo que los formalismos impiden y limitan la actuación libre de las personas.

3. Pienso que el actuar impulsivamente puede ser siempre tan válido como actuar reflexivamente.

$\begin{array}{ll}\bigcirc & \bigcirc \\ \bigcirc & \bigcirc \\ 0 & 0\end{array}$

4. Me gusta afrontar la vida espontáneamente y no tener que planificar todo previamente.

5. Es mejor gozar del momento presente que deleitarse pensando en el pasado o en el futuro.

6. Creo que es preciso saltarse las normas muchas más veces que cumplirlas.

7. En conjunto, hablo más que escucho.

8. Me gusta buscar nuevas experiencias.

9. Me resulta incómodo tener que planificar y prever las cosas

10. Con frecuencia soy una de las personas que más anima las fiestas 


\begin{tabular}{lllll}
\hline $\mathbf{N}^{\circ}$ & ÍTEMS & De acuerdo & Indiferente & En desacuerdo \\
\hline
\end{tabular}

11. Me aburro enseguida con el trabajo metódico y minucioso.

12. Suelo dejarme llevar por mis instintos.

13. Disfruto cuando tengo tiempo para preparar mi trabajo y realizarlo a conciencia.

14. Cuando poseo cualquier información, trato de interpretarla bien antes de manifestar alguna conclusión.

15. Antes de hacer algo estudio con cuidado sus ventajas e inconvenientes.

16. Me gusta analizar y dar vueltas a las cosas.

17. Soy cuidadoso(a) a la hora de sacar conclusiones.

18. Prefiero contar con el mayor número de fuentes de información. Cuanto más datos reúnas para reflexionar, mejor

19. En las discusiones me gusta observar cómo actúan los demás participantes.

20. Prefiero distanciarme de los hechos y observarlos desde otras perspectivas

21. Me gusta considerar diversas alternativas antes de tomar una decisión.

22. Suelo reflexionar sobre los asuntos y problemas.

23. El trabajar a conciencia me llena de satisfacción y orgullo.

24. Normalmente trato de resolver los problemas ordenadamente y paso a paso.

25. Me interesa saber cuáles son los sistemas de valores de los demás y con qué criterios actúan.

26. Estoy a gusto siguiendo un orden en las comidas, en el estudio haciendo ejercicio regularmente.

27. Normalmente encajo bien con personas reflexivas, y me cuesta sintonizar con personas demasiado espontaneas, imprevisibles.

28. Prefiero las cosas estructuradas a las desordenadas.

29. Casi siempre procuro ser coherente con mis criterios y sistemas de valores. Tengo principios y los sigo.

30. Tiendo a ser perfeccionista.

31. Detecto frecuentemente la inconsistencia y puntos débiles en las argumentaciones de los demás.

32. Estoy convencido/a que debe imponerse la lógica y el razonamiento.

33. Siempre trato de conseguir conclusiones e ideas claras

34. Con frecuencia miro hacia adelante para prever el futuro. 


$\mathrm{N}^{\circ} \quad$ ÍTEMS

35. Me molesta las personas que no actúan con lógica.

36. Ante los acontecimientos trato de descubrir los principios y teorías en que se basan.

37. Si trabajo en grupo procuro que se siga un método y un orden.

38. Tengo fama de decir lo que pienso claramente y sin rodeos.

39. Creo que lo más importante es que las cosas funcionen.

40. Me gustan más las personas realistas y concretas que las teóricas.

41. Me atrae experimentar y practicar las últimas técnicas y novedades.

42. Juzgo con frecuencia las ideas de los demás por su utilidad.

43. En las reuniones apoyo las ideas prácticas y realistas.

44. Pienso que debemos llegar pronto al grano, al meollo de los temas.

45. Compruebo antes si las cosas funcionan realmente.

46. Rechazo las ideas originales y espontáneas si no las veo prácticas.

47. Creo que el fin justifica los medios en muchos casos.

48. No me importa hacer todo lo necesario para que sea efectivo mi trabajo.

\section{De acuerdo Indiferente En desacuerdo}

O $\quad 0$

$\bigcirc$

$\bigcirc$

$\bigcirc$

$\bigcirc$

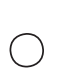

$\bigcirc$

$\bigcirc$

$\bigcirc$

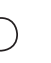

$\bigcirc$

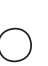

$\bigcirc$

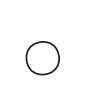

Fecha de recepción: 5 de junio, 2012

Fecha de aceptación: 13 de octubre, 2012 